Development of THGEM-based photon detectors for Cherenkov Imaging Counters

This article has been downloaded from IOPscience. Please scroll down to see the full text article.

2010 JINST 5 P03009

(http://iopscience.iop.org/1748-0221/5/03/P03009)

The Table of Contents and more related content is available

Download details:

IP Address: 137.138.125.144

The article was downloaded on 06/04/2010 at 15:45

Please note that terms and conditions apply. 
$1^{\text {st }}$ International Conference on Micro Pattern Gaseous Detectors, JUNE 12-15, 2009

Kolympari, Crete, Greece

\section{Development of THGEM-based photon detectors for Cherenkov Imaging Counters}

M. Alexeev ${ }^{a}{ }^{M}$ M. Alfonsi, ${ }^{b}$ R. Birsa,${ }^{c}$ F. Bradamante, ${ }^{d}$ A. Bressan, ${ }^{d}$ M. Chiosso, ${ }^{e}$ P. Ciliberti, ${ }^{d}$ G. Croci, ${ }^{b}$ M.L. Colantoni, ${ }^{f}$ S. Dalla Torre,${ }^{c}$ O. Denisov, ${ }^{f}$ S. Duarte Pinto, ${ }^{b}$ V. Duic, ${ }^{d}$ A. Ferrero, ${ }^{e}$ M. Finger, ${ }^{g}$ M. Finger Jr, ${ }^{g}$ H. Fischer,${ }^{h}$ G. Giacomini, ${ }^{i}$ M. Giorgi, ${ }^{d}$ B. Gobbo,${ }^{c}$ R. Hagemann, ${ }^{h}$ F.H. Heinsius, ${ }^{h}$ F. Herrmann, ${ }^{h}$ V. Jahodova,${ }^{j}$ K. Königsmann, ${ }^{h}$ D. Kramer,${ }^{j}$ L. Lauser,${ }^{h}$ S. Levorato, ${ }^{d}$ A. Maggiora, ${ }^{f}$ A. Martin, ${ }^{d}$ G. Menon, ${ }^{c}$ A. Mutter,${ }^{h}$ F. Nerling, ${ }^{h}$ D. Panzieri, ${ }^{a}$ G. Pesaro,,${ }^{d}$ J. Polak,,${ }^{c j}$ E. Rocco, ${ }^{a}$ L. Ropeleswki, ${ }^{b}$ F. Sauli, ${ }^{k}$ G. Sbrizzai, ${ }^{d}$ P. Schiavon, ${ }^{d}$ C. Schill, ${ }^{h}$ S. Schopferer,${ }^{h}$ M. Slunecka, ${ }^{g}$ F. Sozzi, ${ }^{d}$ L. Steiger,${ }^{j}$ M. Sulc, ${ }^{j}$ S. Takekawa, ${ }^{d}$ F. Tessarotto ${ }^{c, 1}$ and H. Wollny ${ }^{h}$

\footnotetext{
${ }^{a}$ INFN, Sezione di Torino and University of East Piemonte, Alessandria, Italy

${ }^{b}$ CERN, European Organization for Nuclear Research, Geneva, Switzerland

${ }^{c}$ INFN, Sezione di Trieste, Trieste, Italy

${ }^{d}$ INFN, Sezione di Trieste and University of Trieste, Trieste, Italy

${ }^{e}$ INFN, Sezione di Torino and University of Torino, Torino, Italy

${ }^{f}$ INFN, Sezione di Torino, Torino, Italy

${ }^{g}$ Charles University, Praga, Czech Republic and JINR, Dubna, Russia

${ }^{h}$ Universität Freiburg, Physikalisches Institut, Freiburg, Germany

${ }^{i}$ INFN, Sezione di Trieste and University of Bari, Bari, Italy

${ }^{j}$ Technical University of Liberec, Liberec, Czech Republic

${ }^{k}$ TERA Foundation, Novara, Italy

E-mail: fulvio.tessarotto@ts.infn.it
}

ABSTRACT: The development of a large size gaseous detector of single photons, able to stably operate at high gain and high rate, and to provide good time resolution and insensitivity to magnetic field would be beneficial to future Cherenkov Imaging Counters. The detector could be based on the use of a multilayer architecture of THGEM electron multipliers coupled to a solid state CsI photocathode. A systematic study of the response of THGEM-based counters versus the geometrical

\footnotetext{
${ }^{1}$ Corresponding author.
} 
parameters has been performed and the main results will be presented. Small photon detector prototypes have been built and preliminary data obtained detecting single photoelectrons are presented as well. The key aspect of photoelectron extraction from the photocathode is illustrated presenting both simulation and dedicated measurement results.

KEYWORDS: Cherenkov detectors; Micropattern gaseous detectors (MSGC, GEM, THGEM, RETHGEM, MICROMEGAS, InGrid, etc); Electron multipliers (gas); Photon detectors for UV, visible and IR photons (gas) (gas-photocathodes, solid-photocathodes) 


\section{Contents}

1 Introduction 1

2 The THGEM electron multiplier 2

3 THGEM production procedures $\quad 2$

4 THGEM characterization $\quad 3$

5 Detection of single photons 6

$\begin{array}{llr}6 & \text { Conclusion } & 8\end{array}$

\section{Introduction}

The photon detectors used so far in Ring Imaging CHerenkov (RICH) counters are either vacuumbased ones, namely photomultiplier tubes in standard or hybrid version, or gaseous detectors coupled to photon converters. The latter represent the only available option for instrumenting very large surfaces at affordable costs.

Gaseous photon detectors have been used in the first generation of RICH counters (E605 [1], DELPHI [2], OMEGA [3] SLD [4], etc.) with photoconverting vapors added to the detector gas mixture, while in the second generation of RICH counters they have mostly been coupled to a solid state photoconverter (HADES [5], COMPASS [6], STAR [7], JLab-HallA [8], ALICE [9], etc.). These second generation detectors have been developed within the RD26 research programme [10] and consist in MWPCs where a cathode plane is formed by a PCB segmented in pads and coated with a CsI film: they represent a successful, consolidated technology.

In spite of their remarkable performances, MWPCs with CsI photocathodes suffer from some limitations: they have to be operated at moderate gain to avoid electrical instabilities accompanied by long recovery time (about 1 day) [6], and they experience a severe decrease of the quantum efficiency caused by aging after accumulating a collected charge of some $\mathrm{mC} / \mathrm{cm}^{2}[11]$. These limitations are related to the ion bombardment of the CsI photocathode and to the presence of an important photon feedback due to the open geometry of the detector.

New detector architectures can overcome these limitations: for instance in a multilayer structure of electron multipliers no secondary photons can reach the photocathode and a good fraction of the ions can be trapped in the intermediate layers. The threshold Cherenkov counter Hadron Blind Detector (HBD) [12] of the PHENIX experiment represents the first application of these ideas: the photon detectors are triple GEM counters operated at low total gain (about 5000). For future RICH applications, however, larger gains are needed because high single photoelectron detection efficiency is mandatory. 
In view of a possible COMPASS RICH upgrade, a dedicated R\&D project [13] was started, aimed to investigate the possibility of building a large size gaseous detector of single photons, able to stably operate at large gain, at high rate, and to provide fast response, good time resolution and insensitivity to magnetic field.

Since the characteristics of THick GEM (THGEM) [14, 15] electron multipliers seemed to cope with the requirements mentioned above, a systematic study of their performances was initiated, in order to optimize their design and production parameters. Detector prototypes based on this technology have been built and tested.

This article describes the status of the R\&D project to develop a THGEM-based photon detector for RICH applications.

\section{The THGEM electron multiplier}

THGEMs are electron multipliers derived from the GEM design, scaling the geometrical parameters and changing the production technology: the $\mathrm{Cu}$-coated kapton foil of the GEM multipliers is replaced by standard PCBs and the holes are produced by mechanical drilling. The rim, a metalfree clearance ring surrounding the hole, is produced by $\mathrm{Cu}$ etching (see figure 1) and corresponds to the uncoated polyamide ring around the GEM holes, due to their conical shape.

The THGEM geometrical parameters cover wide ranges; typical values are: PCB thickness from $0.4 \mathrm{~mm}$ to $1 \mathrm{~mm}$, hole diameter between $0.2 \mathrm{~mm}$ and $1.0 \mathrm{~mm}$, hole pitch from $0.7 \mathrm{~mm}$ to $1.5 \mathrm{~mm}$ and rim width between 0 (no rim) and $0.12 \mathrm{~mm}$.

Large gains have been reported for detectors with single or double THGEM layers [14], as well as good rate capabilities. THGEMs can be produced in large series and large size with standard PCB technology, they have intrinsic mechanical stiffness and are robust against damages produced by electrical discharges. On the other hand, due to the technology used, THGEM-based detectors cannot be built with very low material budget and cannot offer as good a space resolution as GEMbased detectors can.

THGEMs could be used for various detector applications: as active elements of sampling calorimeters or muon trackers, for instance, and in general when wide areas need to be instrumented and space resolution in the $\mathrm{mm}$ range is acceptable. The small gaps between the multiplication stages allow these detectors to be used in magnetic fields.

THGEMs are suited for large surface photon detectors too, because the robustness, the standard production technique and the mechanical characteristics are clear advantages, while the material budget and resolution aspects do not represent a limitation. A THGEM-based photon detector consists in a structure of double or triple THGEM layers, where the first layer is coated with a CsI film and acts as a reflective photocathode. This configuration is preferred to architectures with semitransparent photocathode, as it allows a larger photoconversion rate.

\section{THGEM production procedures}

As a first step, a comparison of different THGEM production procedures was performed: a set of many small size ( $30 \mathrm{~mm} \times 30 \mathrm{~mm}$ active area) THGEM samples has been produced using 5 different methods and several different rim widths up to $100 \mu \mathrm{m}$, including samples with no rim. 

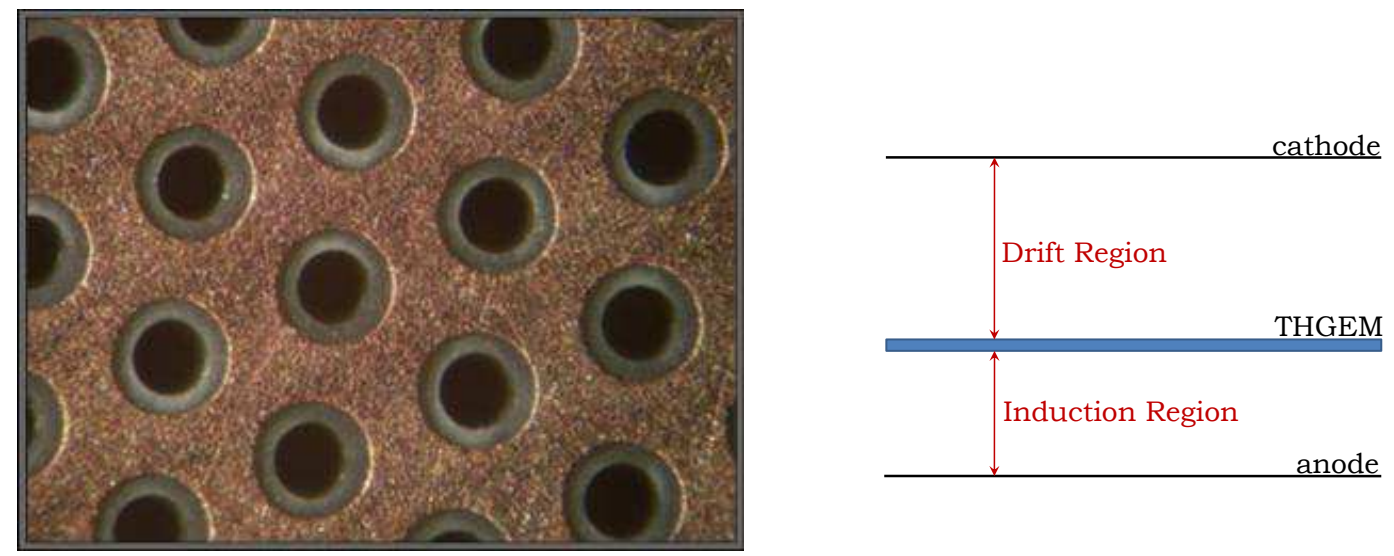

Figure 1. Image of a THGEM with holes of $0.3 \mathrm{~mm}$ diameter, pitch of $0.7 \mathrm{~mm}$ and rim size of $0.1 \mathrm{~mm}$.

Figure 2. Scheme of the THGEM test arrangement.

In the original procedure [14] the rim is formed with standard PCB lithographic image transfer and etching techniques before drilling. For small rims this approach is not suited because it results in excessively off-centered rims; a production technique without image transfer has been used at CERN: a photoresistive layer is applied before drilling; then the etching removes the copper from the unprotected surface created by drilling at the hole edges, producing a rim with an uniform width around the hole.

Three alternative techniques for the rim production have been used by the ELTOS Company ${ }^{1}$ for the THGEM samples. The first one consists in a pure mechanical process, requiring a triple drilling procedure, and the resulting rim surface is inclined by about 120 degrees with respect to the PCB surface. The second one consists in drilling as first step; then, a photoresistive layer is applied and image transfer is performed using the holes as position reference, resulting in well centered rims. The third, called global etching, is a simplified version of the CERN procedure, in which no photoresistive layer is used: after drilling, etching attacks the copper layers at the hole edges and at the surface; this approach is adequate to produce small rims only: about $20 \mu \mathrm{m}$ or less; when large THGEMs have to be produced, the global etching is still possible but the standard etching has to be replaced with micro-etching techniques to achieve good uniformity.

The different THGEM samples have been tested to measure, in nitrogen atmosphere and without radiation sources, the maximum voltage that can be applied between the two PCB faces, before a regime of frequent discharges is observed. As expected, the maximum voltage strongly depends on the rim size and substantially higher voltage can be applied to large rim samples. Comparing samples with the same rim size, some dependence of the maximum voltage on the production technique is clearly seen and the samples produced with the global etching procedure give the best results.

\section{THGEM characterization}

Understanding the role of the various geometrical parameters of the THGEM multipliers has been the next goal of the R\&D activity: more than 30 different THGEM prototypes have been character-

\footnotetext{
${ }^{1}$ ELTOS S.p.A., San Zeno E-44, I-52040 Arezzo (Italy), http://www.eltos.it/.
} 


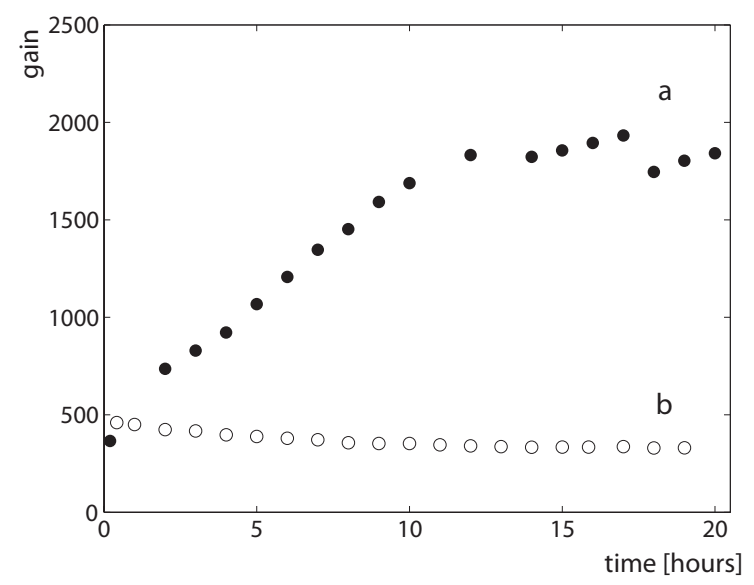

Figure 3. Gain behavior versus time for two THGEMs with the following geometry: thickness $0.4 \mathrm{~mm}$, pitch $0.8 \mathrm{~mm}$ and hole diameter $0.4 \mathrm{~mm}$ (common parameters); different parameter: $0.1 \mathrm{~mm}$ rim for (a), no rim for (b). Continuous detector irradiation; $\Delta \mathrm{V}$ : $1750 \mathrm{~V}$ for (a) and $1330 \mathrm{~V}$ for (b).
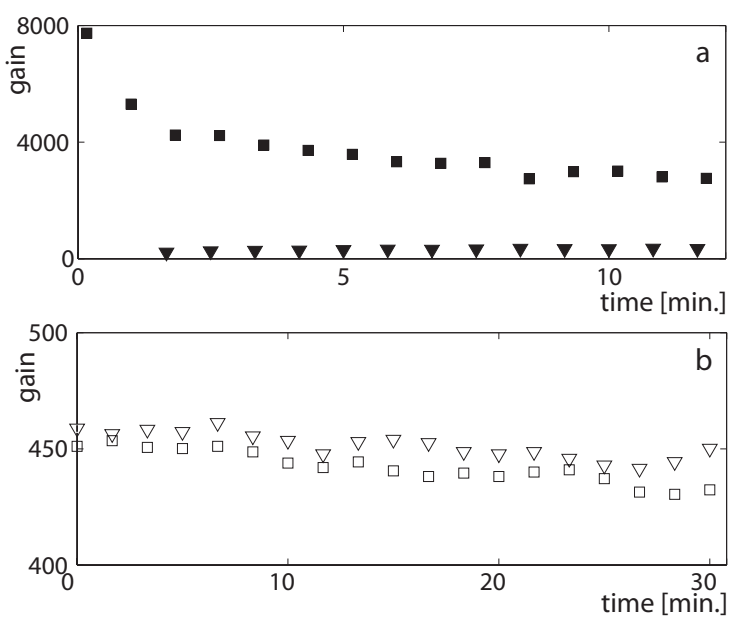

Figure 4. Gain versus time for the THGEMs (a) and (b) described in figure 3. Full (empty) square points represent the gain measured irradiating the THGEM with large (no) rim after it has been for $10 \mathrm{~h}$ at nominal voltage without irradiation. Full (empty) triangle points represent the gain measured irradiating the THGEM with large (no) rim immediately after switching on the high voltage, after it has been switched off for 1 day.

ized using soft X-ray sources and a standard, non flammable gas mixture $\left(\operatorname{Ar}: \mathrm{CO}_{2}=70: 30\right)$. The samples to be tested are mounted in single layer mode between two electrodes in the configuration sketched in figure 2 .

A multiplication voltage $\Delta \mathrm{V}$ is applied between the two THGEM faces; an electric field $\mathrm{E}_{d r i f t}$ is established in the region above the top THGEM face (lower potential), in order to focus the ionization electrons through the THGEM holes; it is obtained applying to the cathode electrode a potential lower than the one of the top THGEM face. An electric field $\mathrm{E}_{\text {induction }}$ is applied in the region below the bottom THGEM face (higher potential) to guide part of the electrons created in the multiplication process to the anode electrode.

Amplitude spectra of the anode signals are collected and the currents absorbed by each electrode (cathode, THGEM top, THGEM bottom, anode) are measured. To perform the latter exercise, cheap and low consumption current meters with $\sim 1 \mathrm{pA}$ resolution have been designed and built: they are powered via batteries and carefully isolated in order to operate at high voltage.

The characterization protocol includes a set of measurements in different conditions, to find the optimal configurations of the $\mathrm{E}_{\text {drift }}$ and $\mathrm{E}_{\text {induction }}$ fields and the detector response (currents, effective gain and energy resolution) for different multiplication voltages. Long term (days) measurements of the detector gain stability are performed for each sample.

The most relevant results are summarized in figures 3 to 6 . Large gains can be obtained multiplying electrons by THGEM with large rim (about $0.1 \mathrm{~mm}$ ) but the gain stability in time strongly depends on the rim size. Gain variations $\leq 20 \%$ are observed when the rim is absent, while huge variations are clearly observed when the rim is large $(0.1 \mathrm{~mm})$ (figure 3$)$. These variations depend 

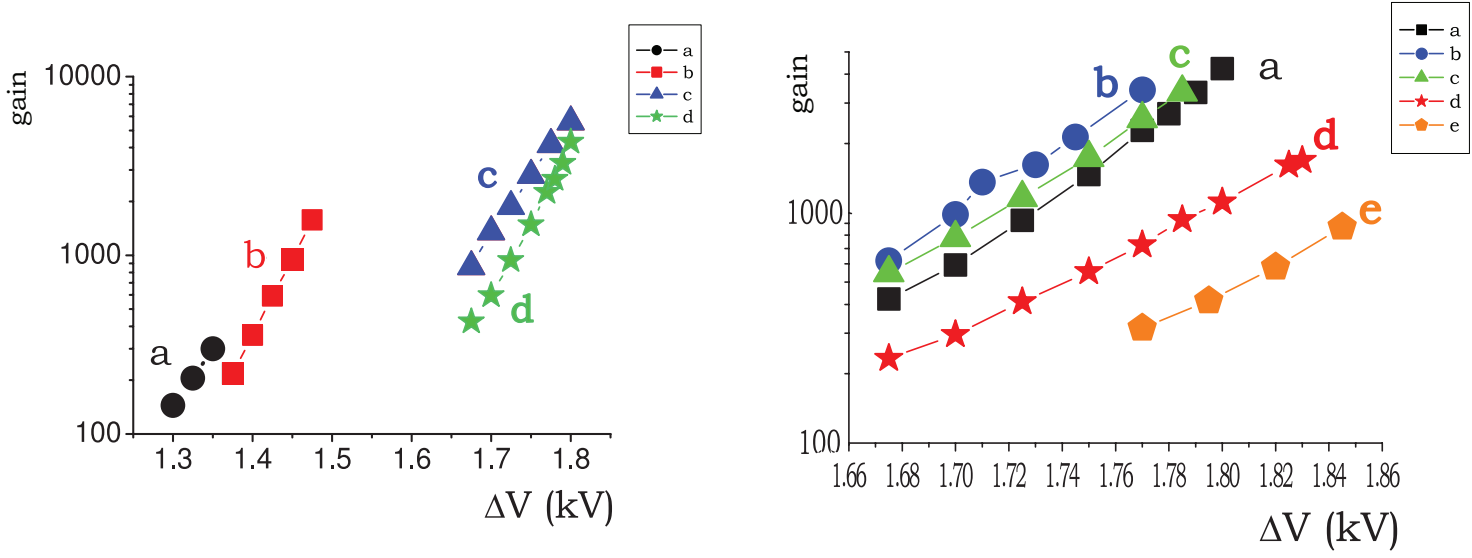

\begin{tabular}{|c|c|c|}
\hline THGEM & Diameter (mm) & Thickness (mm) \\
\hline $\mathbf{a}$ & 0.0 & 0.4 \\
\hline b & 0.01 & 0.4 \\
\hline c & 0.1 & 0.4 \\
\hline d & 0.0 & 0.6 \\
\hline
\end{tabular}

Figure 5. Effective gain versus applied $\Delta \mathrm{V}$ for THGEMs with holes of $0.3 \mathrm{~mm}$ diameter and 0.7 $\mathrm{mm}$ pitch, having different rim widths and thicknesses.

\begin{tabular}{|c|c|c|}
\hline THGEM & Diameter $(\mathbf{m m})$ & Pitch $(\mathbf{m m})$ \\
\hline $\mathbf{a}$ & 0.3 & 0.7 \\
\hline $\mathbf{b}$ & 0.3 & 0.6 \\
\hline $\mathbf{c}$ & 0.3 & 0.5 \\
\hline $\mathbf{d}$ & 0.4 & 0.7 \\
\hline e & 0.5 & 0.7 \\
\hline
\end{tabular}

Figure 6. Effective gain versus applied $\Delta \mathrm{V}$ for THGEMs with $0.6 \mathrm{~mm}$ thickness and no rim, having different hole diameters and pitches.

on the irradiation rate when the detector is powered. In figure 4(a) the short time gain variations of a THGEM with large rim are presented for two extreme cases: irradiating for a few minutes after the THGEM has been for 10 hours at nominal voltage without irradiation, and irradiating for a few minutes at voltage switch-on after 1 day with no voltage. Large variations in the measured gain and totally different behaviors are seen. For a THGEM with no rim (figure 4(b)) the same measurements show on the contrary that the gain is almost independent of the previous $\Delta \mathrm{V}$ and irradiation history.

This behavior is interpreted as caused by the displacement of charges in the PCB fiberglass plate when the high voltage is applied. This phenomenon is slow (it takes hours or days) and the resulting charge distribution is not screened in the rim region. When the detector is irradiated, the effective field causing the dielectric polarization is partially screened by the accumulation of charged particles (ions or electrons) on the free dielectric surface. The different irradiation rate thus results in different charge distribution and, consequently, in different detector gain.

In figure 5 the effective gain as function of the multiplication voltage $\Delta \mathrm{V}$ is displayed for THGEM samples with different rim and thickness: the maximum stably attainable gain increases with the rim size and with the PCB thickness.

Figure 6 illustrates the dependence of the gain on other geometrical parameters: the hole diameter plays a major role (larger gain is observed using smaller diameters) while the pitch has a mild influence on the effective gain.

For a subset of the characterized THGEMs the gain versus rate has been measured too: using soft X-rays (releasing about 300 ion pairs per conversion) no rate dependence of the gain up to rates exceeding $100 \mathrm{kHz} / \mathrm{mm}^{2}$ has been observed for THGEMs without rim, while some dependence is seen with large rims. 


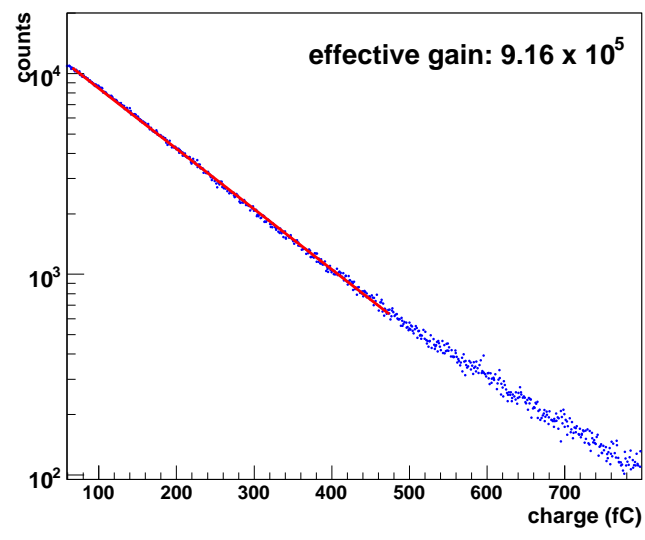

Figure 7. Single photoelectron amplitude spectra measured with the THGEM detector described in the text.

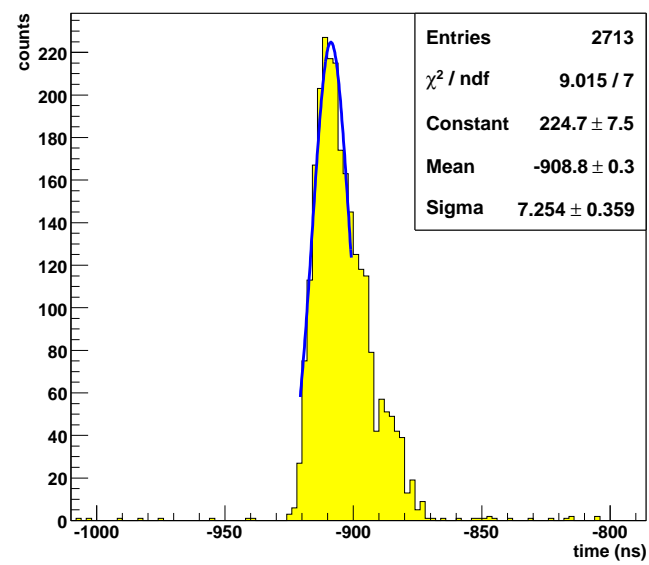

Figure 8. Measured time spectrum for single tagged photon signals from a triple THGEM chamber.

This characterization exercise clearly indicates that small rim sizes (between 5 and $30 \mu \mathrm{m}$ ) are preferable in order to guarantee stable detector response, in spite of the enhanced gain performance obtained with large rim THGEMs; on the other hand, the etching procedure applied to produce a rim is beneficial, as the hole edges are smothered and THGEMs with a rim can stand higher voltages than those with no rim.

\section{Detection of single photons}

Small photon detector prototypes have been built and operated with various configurations of multiple THGEMs, always with CsI coating on the top of the first THGEM. Several tests have been performed using either continuous $\left(\mathrm{D}_{2}\right)$ UV lamp or pulsed UV LED source with $600 \mathrm{ps}$ long light pulses, ${ }^{2}$ with attenuated light in order to establish the single photoelectron condition. The anodic electrode of the detector is a PCB segmented in 16 pads, allowing either analog or digital (16 channels) readout to be mounted.

The most extensively used prototype detector consists in a triple-layer configuration of identical THGEMs having $0.4 \mathrm{~mm}$ thickness, $0.8 \mathrm{~mm}$ pitch, $0.4 \mathrm{~mm}$ hole diameter and $10 \mu \mathrm{m}$ rim; it is operated with various gas mixtures, including methane-rich argon mixtures.

A typical single photoelectron spectrum obtained with this prototype detector and a gas mixture of $\mathrm{Ar}: \mathrm{CH}_{4}=50: 50$ is shown in figure 7: effective gains around $10^{6}$ are recently being routinely obtained in laboratory tests. A typical time spectrum for single photon signals, obtained using the electronic chain described in [16] and, in particular, the F1 TDC [17] with 108 ps bin size, is shown in figure 8: a time resolution close to $7 \mathrm{~ns}$ is obtained from the fit of the main component of the time spectrum. Further studies to investigate the characteristics of the time response in different conditions are foreseen.

\footnotetext{
${ }^{2}$ obtained powering an UV LED by the PDL $800-B$ pulsed power supply by PicoQuant GmbH, Berlin, Germany.
} 


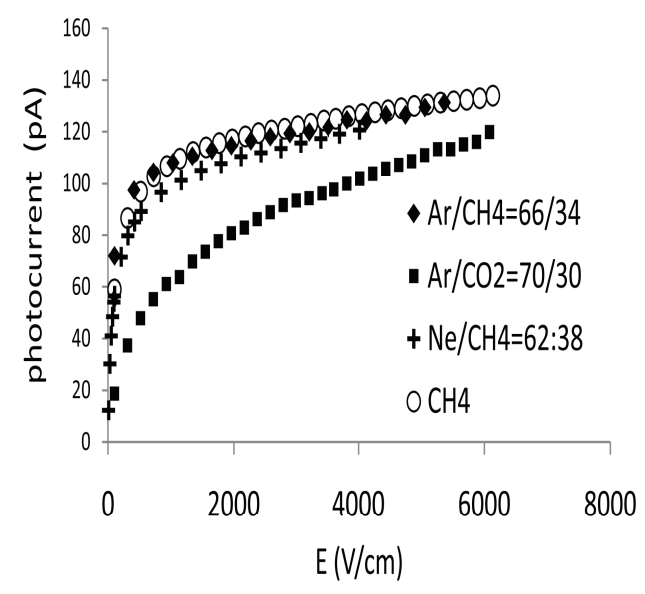

Figure 9. CsI photocurrent versus applied field in various gas and gas mixtures at atmospheric pressure; systematic uncertainty at the $1 \%$ level.

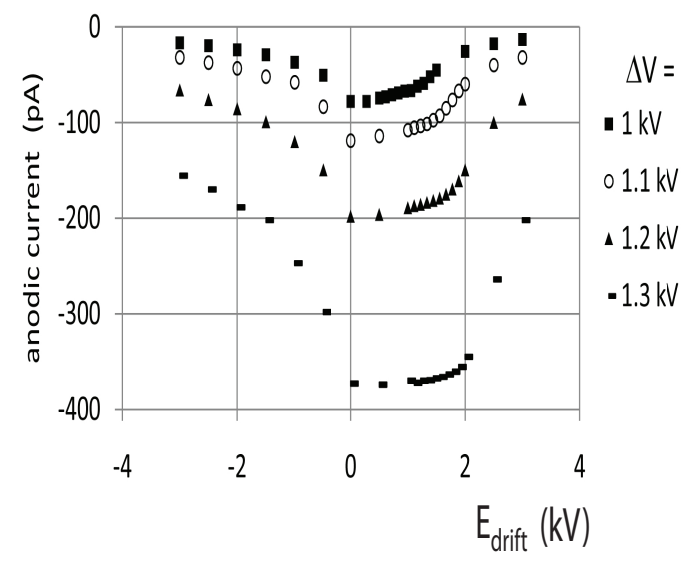

Figure 10. Anodic current measured in a single THGEM detector with CsI reflective photocathode versus the additional electric field applied; the different point sets have been obtained for different values of the potential $\Delta \mathrm{V}$ applied between the THGEM faces.

The photoelectron extraction yield obtainable in the proposed detector architecture has been investigated. As a preliminary step, a set of reference measurements of photocurrent as function of the electric field value at the CsI photocathode in various gases and at different gas pressures has been revisited. Figure 9 shows some of the results obtained. It can be noticed that for the gases and gas mixtures with the best performance, the backscattering phenomenon limiting the extraction yield is overcome only for electric field values above $1 \mathrm{kV} / \mathrm{cm}$.

In a THGEM-based detector, the field at the photocathode surface is the combination of the electric field due to the potential applied between the two THGEM faces, called dipole field since it has a shape similar to the sum of dipole fields (one dipole per hole), and the drift field due to the potential between the photocathode surface and the wire cathode electrode facing it. Electron trajectory simulations indicate that when the drift field is negative (it points toward the photocathode), the wire cathode, being more positive than the THGEM photocathode, collects part of the photoelectrons, which are then lost. For zero drift field, all the electron trajectories enter the holes, but when the drift field is positive (oriented toward the wire cathode), the resulting field at the photocathode surface is reduced, and so is the probability of photoelectron extraction, and the field may even become too feeble to efficiently guide the photoelectrons into the THGEM holes. The lower is the dipole field, the smaller is the critical drift field that causes a reduction of the photoelectron collection efficiency. The behavior predicted by the simulations is confirmed by measurements using a single THGEM layer with CsI coating, a UV lamp and measuring the current collected at the detector anode. The plots in figure 10 clearly indicate a sharp current decrease when the drift field is negative, a rough plateau for moderate values of positive drift field, followed by a drop when the total field becomes too low. In particular, the current drops at lower values of the drift field when the potential applied between the THGEM faces is smaller, namely for lower dipole field as expected from the simulation. 


\section{Conclusion}

In view of the development of a THGEM-based photon detector for RICH applications more than 30 THGEMs with different geometrical and production parameters have been characterized in a systematic way. This allowed to gain insight in the role of the geometrical parameters and to identify values which are suited for the envisaged application.

Small prototypes of photon detectors have been built and operated in the laboratory at gains close to $10^{6}$. The most relevant parameters for the optimization the photoelectron extraction are being studied using both simulations and measurements with reflective CsI photocathodes deposited on small THGEMs.

The optimization of the detector response and the engineering problems related to larger size prototype building are presently being investigated and test beam exercises are scheduled.

\section{Acknowledgments}

The authors are grateful to Prof. A. Breskin for his constant encouragement and many stimulating discussions. This work has partly been performed in the framework of the RD51 collaboration.

\section{References}

[1] M. Adams et al., $\pi / K / p$ Identification with a Large Aperture Ring Imaging Cherenkov Counter, Nucl. Instrum. Meth. 217 (1983) 237.

[2] E. Albrecht et al., Operation, optimisation and performance of the DELPHI RICH detectors, Nucl. Instrum. Meth. A 433 (1999) 47 and references therein.

[3] H.W. Siebert et al., The Omega RICH, Nucl. Instrum. Meth. A 343 (1994) 60.

[4] CRID collaboration, J. Va'vra, Long-term operational experience with the barrel CRID at SLD, Nucl. Instrum. Meth. A 433 (1999) 59.

[5] J. Friese et al., Enhanced quantum efficiency for CsI grown on a graphite- based substrate coating, Nucl. Instrum. Meth. A 438 (1999) 86;

H. Rabus et al., Quantum efficiency of cesium iodide photocathodes in the 120-220 nm spectral range traceable to a primary detector standard, Nucl. Instrum. Meth. A 438 (1999) 94;

R. Gernhauser et al., Cesium iodide photon converter performance in a gaseous RICH detector, Nucl. Instrum. Meth. A 438 (1999) 104.

[6] E. Albrecht et al., Status and characterisation of COMPASS RICH-1, Nucl. Instrum. Meth. A 553 (2005) 215 and references therein.

[7] STAR collaboration, A. Braem et al., Identification of high $p_{T}$ particles with the STAR-RICH detector, Nucl. Instrum. Meth. A 499 (2003) 720.

[8] F. Garibaldi et al., A proximity focusing RICH detector for kaon physics at Jefferson Lab Hall A, Nucl. Instrum. Meth. A 502 (2003) 117;

M. Iodice et al., Performance and results of the RICH detector for kaon physics in Hall A at Jefferson Lab, Nucl. Instrum. Meth. A 553 (2005) 231.

[9] ALICE collaboration, Technical Design Report of the High Momentum Particle Identification Detector, CERN-LHCC-98-019, ALICE-TDR-1, 14 August 1998; 
A. Braem et al., Identification of high $p \perp$ particles with the STAR-RICH detector, Nucl. Instrum. Meth. A 499 (2004) 720.

[10] RD26 collaboration,RD26 status reports, CERN-DRDC-93-36, CERN-DRDC-94-49, CERN-DRDC-96-20.

[11] A. Braem et al., Results from the ageing studies of large CsI photocathodes exposed to ionizing radiation in a gaseous RICH detector, Nucl. Instrum. Meth. A 553 (2005) 187;

H. Hoedlmoser et al., Long term performance and ageing of CsI photocathodes for the ALICE/HMPID detector, Nucl. Instrum. Meth. A 574 (2007) 28.

[12] A. Kozlov et al., Development of a triple GEM UV-photon detector operated in pure CF-4 for the PHENIX experiment, Nucl. Instrum. Meth. A 523 (2004) 345 [physics/0309013];

Z. Fraenkel et al., A hadron blind detector for the PHENIX experiment at RHIC, Nucl. Instrum. Meth. A 546 (2005) 466 [physics/0502008];

A. Milov et al., Construction and expected performance of the hadron blind detector for the PHENIX experiment at RHIC, J. Phys. G 34 (2007) S701.

[13] M. Alexeev et al., The quest for a third generation of gaseous photon detectors for Cherenkov imaging counters, Nucl. Instrum. Meth. A 610 (2009) 174.

[14] A. Breskin et al., A concise review on THGEM detectors, Nucl. Instrum. Meth. A 598 (2009) 107 [arXiv:0807.2026] and references therein.

[15] J.M. Bidault et al., A Novel UV Photon Detector with Resistive Electrodes, Nucl. Phys. B Proc. Suppl. 158 (2006) 199.

[16] P. Abbon et al., Read-out electronics for fast photon detection with COMPASS RICH-1, Nucl. Instrum. Meth. A 587 (2008) 371.

[17] H. Fischer et al., Implementation of the dead-time free F1 TDC in the COMPASS detector readout, Nucl. Instrum. Meth. A 461 (2001) 507 [hep-ex/0010065]. 